\title{
Surgical management of zygomatic complex fractures in a major trauma centre
}

\author{
Daniel Bradley', Billy Leung', Shobhit Saxena', Mohammed Dungarwalla², David Chapireau², Kathleen \\ Fan $^{2}$
}

'Faculty of Dentistry, Oral \& Craniofacial Sciences, King's College London, London SE1 1UL, United Kingdom. ${ }^{2}$ Department of Oral \& Maxillofacial Surgery, King's College London Hospital NHS Foundation Trust, London SE5 9RS, United Kingdom.

Correspondence to: Dr. Daniel Bradley, Faculty of Dentistry, Oral \& Craniofacial Sciences, King's College London, London SE1 1UL, United Kingdom. E-mail: drdanielrobinbradley@hotmail.com

How to cite this article: Bradley D, Leung B, Saxena S, Dungarwalla M, Chapireau D, Fan K. Surgical management of zygomatic complex fractures in a major trauma centre. Plast Aesthet Res 2019;6:11. http://dx.doi.org/10.20517/2347-9264.2019.06

Received: 27 Jan 2019 First Decision: 21 Apr 2019 Revised: 28 Apr 2019 Accepted: 16 May 2019 Published: 24 May 2019

Science Editor: Raúl González-García Copy Editor: Cai-Hong Wang Production Editor: Jing Yu

\begin{abstract}
Aim: To analyse the epidemiology, aetiology, and surgical management of zygomatic complex (ZMC) fractures in our major trauma centre, and to compare the number and location of fixation points and surgical access in our patient cohort with the literature.
\end{abstract}

Methods: Retrospective analysis of all operative cases (Open Reduction and Internal Fixation) of zygomatic complex fractures over a one year period (2016).

Results: A greater proportion of patients in our cohort (54\%) were treated with one-point fixation compared to the literature, with the zygomaticomaxillary (ZM) buttress being the most popular fixation point (90\%). ZM buttress and frontozygomatic (FZ) suture were the commonest choices for two-point fixations (70\%). Buccal sulcus incision was used for ZM access in all cases. For FZ access, upper blepharoplasty incision was the most common (56\%). For infra-orbital margin access, transconjunctival incision was the most common (75\%). There was no significant association between number of fixation points and presence of associated injuries, impact of injury, or time to operation. There were no post-operative complications.

Conclusion: A greater proportion of patients in our cohort were successfully treated with one point fixation compared to the literature, and fewer patients underwent orbital floor exploration and repair in our cohort compared to the literature. This study highlights the ongoing variation in the surgical management of ZMC fractures.

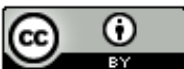

(C) The Author(s) 2019. Open Access This article is licensed under a Creative Commons Attribution 4.0 International License (https://creativecommons.org/licenses/by/4.0/), which permits unrestricted use, sharing, adaptation, distribution and reproduction in any medium or format, for any purpose, even commercially, as long as you give appropriate credit to the original author(s) and the source, provide a link to the Creative Commons license, and indicate if changes were made.

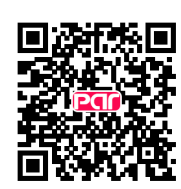


Keywords: Zygomatic complex fractures, ZMC, open reduction internal fixation, zygomaticomaxillary buttress

\section{INTRODUCTION}

Zygomatic complex (ZMC) fractures are relatively common. A literature search showed ZMC fractures to account for approximately $15 \%-23.5 \%$ of maxillofacial fractures ${ }^{[1-3]}$. The incidence of ZMC fractures varies with geographical location, socioeconomic trends, and incidence of road traffic collisions (RTCs), alcohol abuse and drug abuse $\mathrm{e}^{[4]}$. A number of studies had shown ZMC fractures to be the second most common facial fracture, after nasal bone or mandible fractures ${ }^{[3-6]}$. Common causes of ZMC fractures include interpersonal violence (15\%-64.5\%), RTCs (13.9\%-49\%), as well as falls, occupational accidents, and sport-related injuries ${ }^{[3,7,8]}$. Furthermore, ZMC fractures are more common in men than women, and most commonly occur in the third decade of life ${ }^{[2]}$.

An intact zygoma (or zygomatic bone) and its surrounding bony anatomy are essential for maintaining facial contour, such as cheek prominence, as well as orbital integrity ${ }^{[5]}$. Anatomically, the zygoma is attached to the frontal bone (via the frontozygomatic suture), the maxilla (via the zygomaticomaxillary suture), the squamous part of the temporal bone (via the zygomaticotemporal suture) and the sphenoid bone (via the zygomaticosphenoid suture) [Figure 1$]^{[6]}$. Fractures that involve the zygoma often occur at these four suture sites, leading to a "tetrapod" fracture pattern, known as a "zygomatic complex fracture" (ZMC). Furthermore, the zygoma is connected to the maxilla and sphenoid bone as part of the inferior orbital floor, and forms the lateral orbital margin with the frontal bone. Thus, fractures of the zygomatic complex inevitability lead to a certain degree of orbital defect. Other fracture patterns, include isolated zygomatic arch fractures, or ZMC fractures with associated pan-facial fractures, such as Le Fort II and III fracture patterns. Indication for fixation of zygomatic fractures includes aesthetic defects (e.g., cheekbone flattening or a dimple) or functional defects (e.g., restrictive mouth opening, malocclusion or ophthalmic issues such as diplopia, restricted eye movements, enopthalmus and hypoglobus).

There are currently no widely accepted treatment protocols or guidelines on the surgical management of ZMC fractures. The fixation points used in the Open Reduction and Internal Fixation (ORIF) of ZMC fractures are shown in Figure 2. A review of the literature shows that for ORIF of ZMC fractures, the number of fixation points used, their location, as well as the incisional access to these fixation points is variable ${ }^{[1-4]}$. A multidisciplinary survey by Farber et al.$^{[10]}$ in 2016 involving Otorhinolaryngology (ENT), Plastic and Oral and Maxillofacial (OMF) surgeons, demonstrated variable treatment choices for ZMC fractures regarding the location and number of fixation points, surgical approaches, as well as the need for orbital floor exploration. Interestingly, across all three specialties, it was demonstrated that a greater number of fixation points were chosen by surgeons with less than 10 years' experience ${ }^{[10]}$.

With regards to one-point fixation, there is variable support from the literature regarding its efficacy, and there is no consensus regarding the optimum anatomical position for one point fixation between the zygomaticomaxillary (ZM) buttress, the infraorbital margin (IOM) and the frontozygomatic (FZ) region, as well as the optimum surgical access to these anatomical fixation points ${ }^{[4,11,12]}$. The ZM buttress has been quoted to be a popular choice for one-point fixation in some literature, whilst others have quoted the FZ suture as their first choice, but beyond this, there is little consensus ${ }^{[4,11-13]}$. Some literature advocates the fixation of both the IOM and FZ suture for any displaced ZMC fractures, and for cases with displacement greater than $5 \mathrm{~mm}$, the use of 3-point fixation is recommended ${ }^{[13]}$.

\section{METHODS}

We retrospectively retrieved, from an online database, all operative cases (ORIF) of zygomatic complex fractures, isolated zygomatic arch fractures, with and without other associated operative procedures (e.g., 


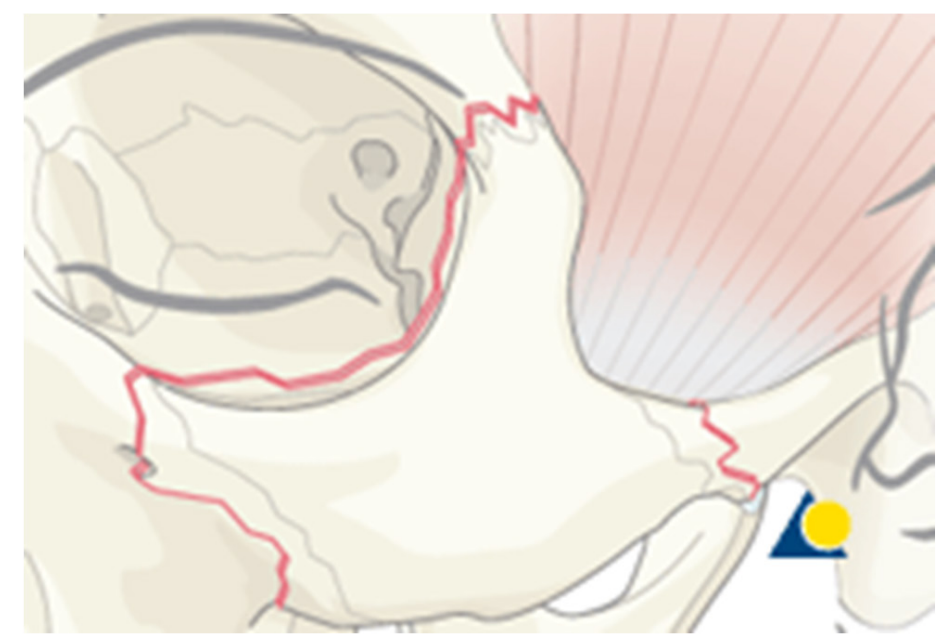

Figure 1. Image reproduced with permission from AO Surgery Reference www.aosurgery.org showing the left zygomatic bone outlined in red, with its anatomical relationship to the frontal bone (superiorly, forming the frontozygomatic suture), maxilla (medially, forming the zygomaticomaxillary suture and infraorbital rim), and squamous part of the temporal bone (laterally, forming the zygomaticotemporal suture), as well as forming part of the orbital floor. Fractures commonly occur at the three above mentioned suture sites, thus result in a classic "tripod fracture"[9]

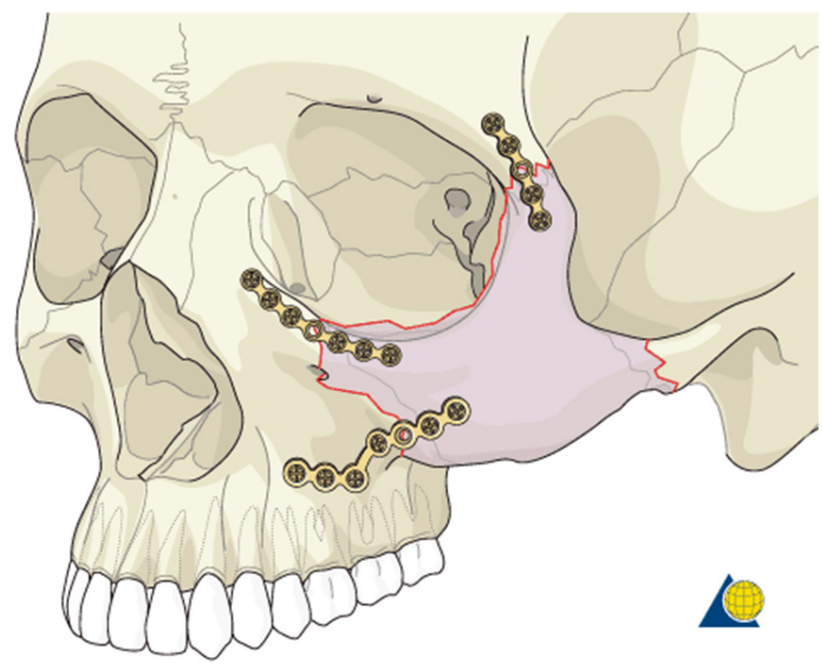

Figure 2. Image reproduced with permission from AO Surgery Reference, www.aosurgery.org illustrating a three-point fixation of a ZMC fracture at the frontozygomatic (FZ) suture (top), infraorbital margin (IOM) (middle), and zygomaticomaxillary (ZM) buttress (bottom)

MUA nose, orbital floor exploration, orbital floor fixation or Le Fort fracture fixations) over a one year period (2016) at our trauma centre, Kings College London Hospital.

Our data set included demographic data (age, sex, relevant past medical history, smoking and alcohol intake status), aetiology (mechanism and impact of injury), treatment timeline (including presentation, referral pathway, time to outpatient clinic and time to operation), clinical features (including head injury, eye signs e.g., enopthalmus, hypoglobus, diplopia, restricted eye movements, infraorbital nerve paraesthesia, aesthetic deficit e.g., cheek flattening, infraorbital rim deformity and functional deficit e.g., restricted mouth opening and malocclusion), diagnosis, type of operation (ORIF, indirect reduction, with or without associated operative procedures, location and number of fixation points, and type of incision used for access), and outcomes and follow up. 

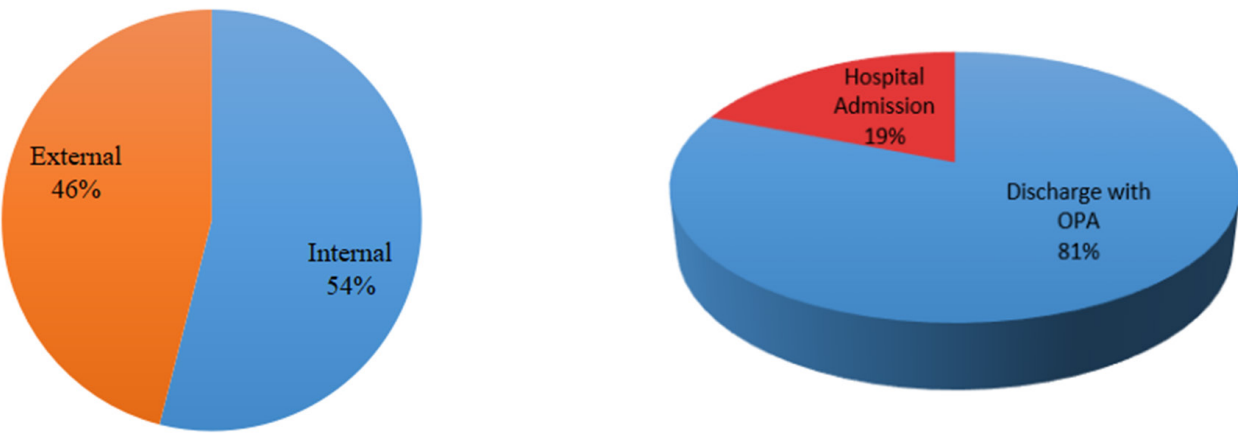

Figure 3. Chart demonstrating the referral pathway and outcome from initial presentation: (left) referral: $55 \%$ cases were internal referrals from our emergency department, and $45 \%$ were from external sources e.g., other hospitals, urgent care centre or general practice; (right) initial outcome: majority of patients were discharged after initial review (81\%), with the remaining being admitted (19\%), often with other associated injuries

All conservatively managed cases of ZMC fractures were excluded. The data was collected and recorded on a shared data-protected Excel spread sheets and was conducted by the author and co-authors. The project was authorised by the Kings College Hospital NHS Foundation Trust, and there was no conflict of interest issues.

\section{RESULTS}

The 2016 cohort consisted of 53 operative cases. There were $40 \mathrm{ZMC}$ fractures and 13 isolated zygomatic arch fractures.

One ZMC fracture case was excluded from the points of fixation and surgical incision analysis. This patient was a polytrauma patient who had surgery delayed by 49 days due to their concurrent injuries, and it was not possible to reduce the ZMC fracture intra-operatively. A bone graft was therefore taken from the anterior maxillary sinus wall and secured to zygomatic body to aesthetically improve the patient's cheek flattening.

\section{Demographics}

Of the operated ZMC fractures in 2016, the mean age was 33.1, median of 30.0, mode of 27 and range was 16-69 years. The majority of cases were male $(89 \%, n=47)$, with $11 \%(n=6)$ female, and $87 \%(n=46)$ were fit and well, with $13 \%(n=7)$ having associated medical comorbidity (including hypertension, asthma, high cholesterol and chronic gastritis).

\section{Referral, presentation and treatment timeline}

Just over half of the cases $(55 \%, n=29)$ were direct internal referrals from our Emergency Department and $45 \%(n=24)$ were external referrals [Figure 3]. The majority $(81 \%, n=43)$ of cases (internal or external referrals) were discharged on initial presentation and arranged for an OMFS outpatients appointment (OPA), $19 \%(n=10)$ were admitted (most commonly under the trauma team) and received inpatient OMFS review [Figure 3]. The mean duration between time of injury to initial presentation was 0.28 days (median 0 , mode 0 , range 0-7), and between initial presentation to outpatient follow up was 7.9 days (median 7.5, mode 5, range 1-19) [Figure 4]. Furthermore, the mean duration between injury to operation was 15.4 days (median 15.5, mode 15, range 0-29), and between operation to discharge was 1 day (median 1, mean 1, range 0-12). The mean duration between discharge and first outpatients follow up was 14 days (median 12, mode 10, range 3-31) [Figure 4].

\section{Aetiology}

The most common cause of ZMC fracture was from interpersonal violence $(53 \%, n=28)$, followed by $23 \%(n=12)$ falls, $13 \%(n=7)$ RTCs. In the remaining six patients, causes included sports injuries and occupational injuries. 


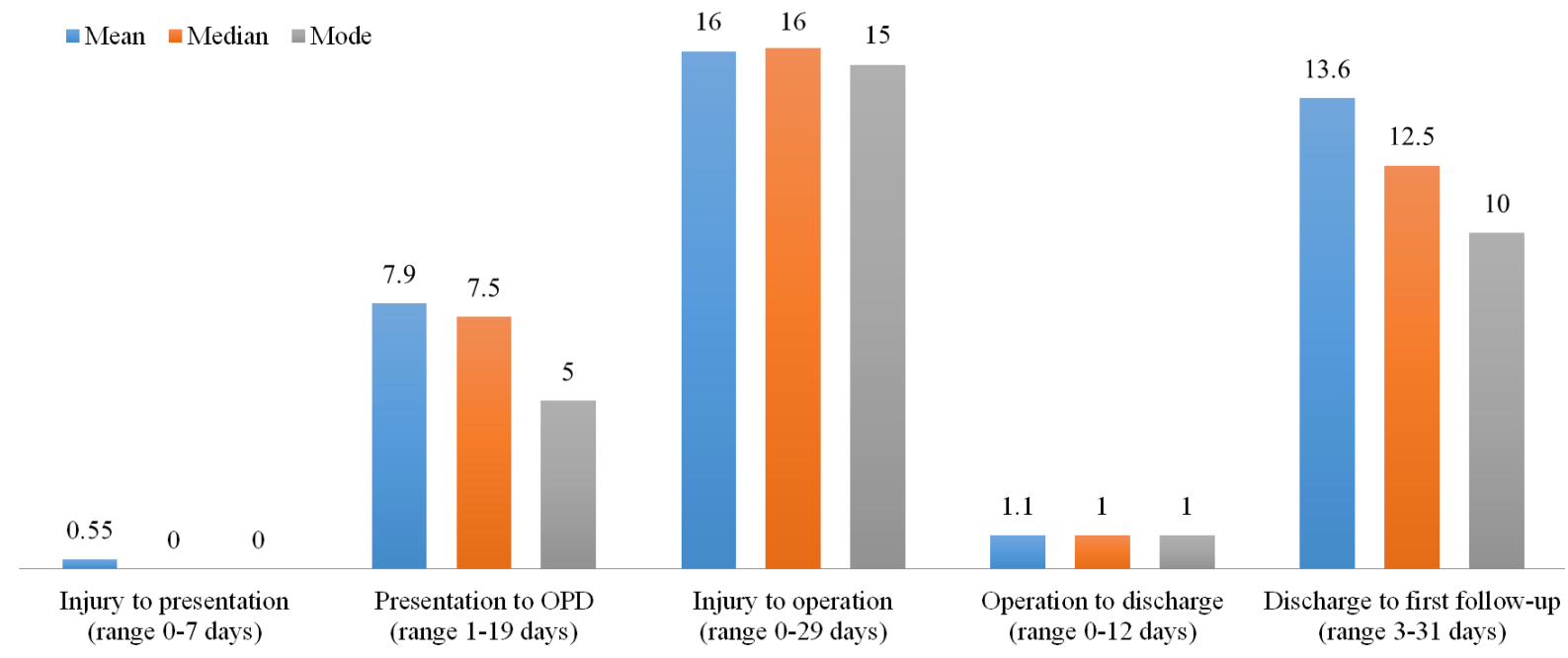

Figure 4. Graph demonstrating the duration (days) between various aspect of the treatment pathway (mean, median, mode and range): injury to initial presentation; initial presentation to outpatient department (OPD) follow-up; injury to time of operation; operation to discharge i.e., length of hospital stay post-op; time between discharge and first follow-up

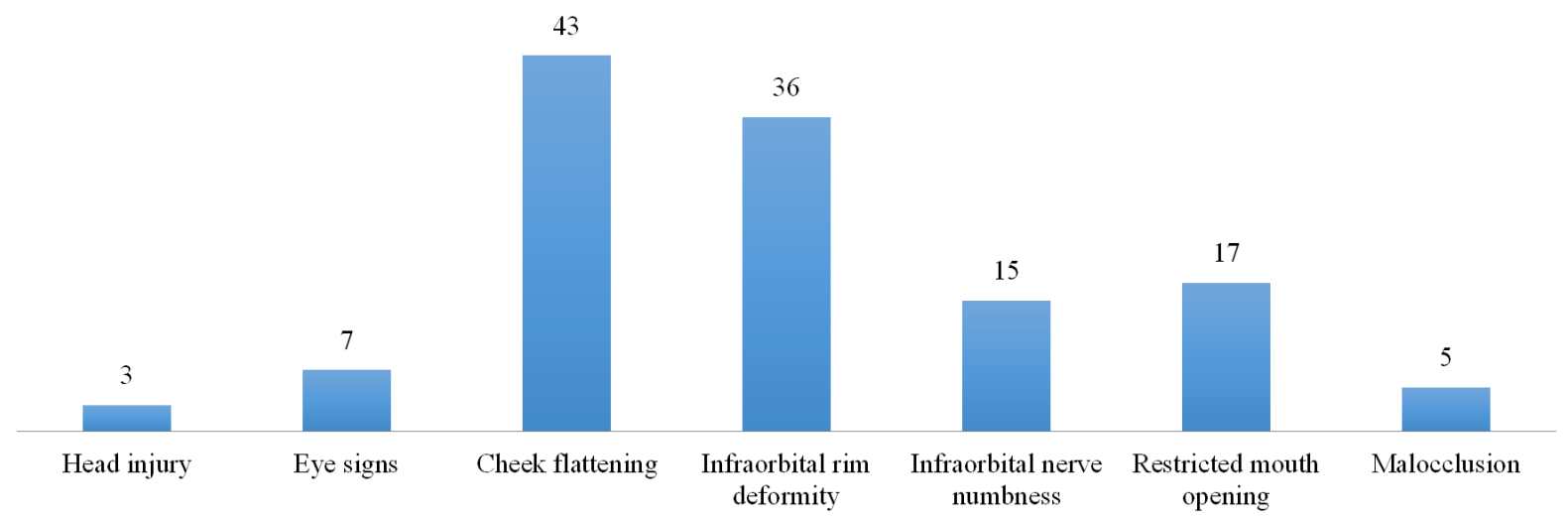

Figure 5. Graph showing the number of patients with particular clinical features in the 2016 cohort $(n=53)$

There was inadequate documentation to determine the possible association of alcohol and/or illicit drug intoxication to the injury. Regarding impact of injury, $81 \%(n=43)$ were low impact, $15 \%(n=8)$ were high impact, and $4 \%(n=2)$ was not recorded. Low impact injuries included punch-related assaults or minor mechanical falls (less than $2 \mathrm{~m}$ in height), compared to RTCs, assaults from hard objects, or falls from a significant height (greater than $2 \mathrm{~m}$ ), which were considered high impact injuries.

\section{Clinical features}

There were $6 \%(n=3)$ of patients who had an associated head injury, $13 \%(n=7)$ with eye signs (e.g., diplopia, enopthalmus, hypoglobus, limitation of eye movements), $81 \%(n=43)$ with flattening of malar prominence $68 \%(n=36)$ with palpable infraorbital step, $28 \%(n=15)$ with infraorbital nerve numbness, $32 \%(n=17)$ with restricted mouth opening and $9 \%(n=5)$ with malocclusion [Figure 5].

\section{Diagnosis}

Of the 53 patients in our 2016 cohort, 55\% $(n=29)$ were isolated ZMC fractures without significant orbital floor defect, 20\% ( $n=11)$ were ZMC fractures with other associated maxillofacial injuries, and 25\% $(n=13)$ were isolated zygomatic arch fractures only [Figure 6]. 
Bar chart showing the number of isolated ZMC fractures, ZMC fractures with associated injuries, and isolated zygomatic arch fractures

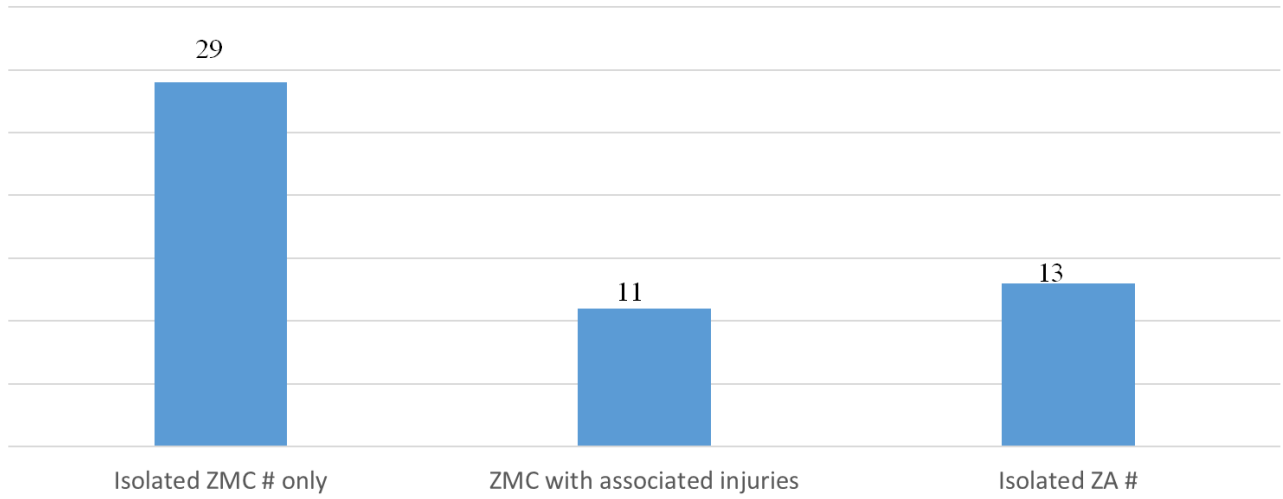

Figure 6. Bar chart showing the number of isolated ZMC fractures, the number of patients who also had associated maxillofacial injuries, and the number of isolated zygomatic arch fractures

Bar chart showing the number of associated maxillofacial injuries in our 2016 ZMC fracture cohort

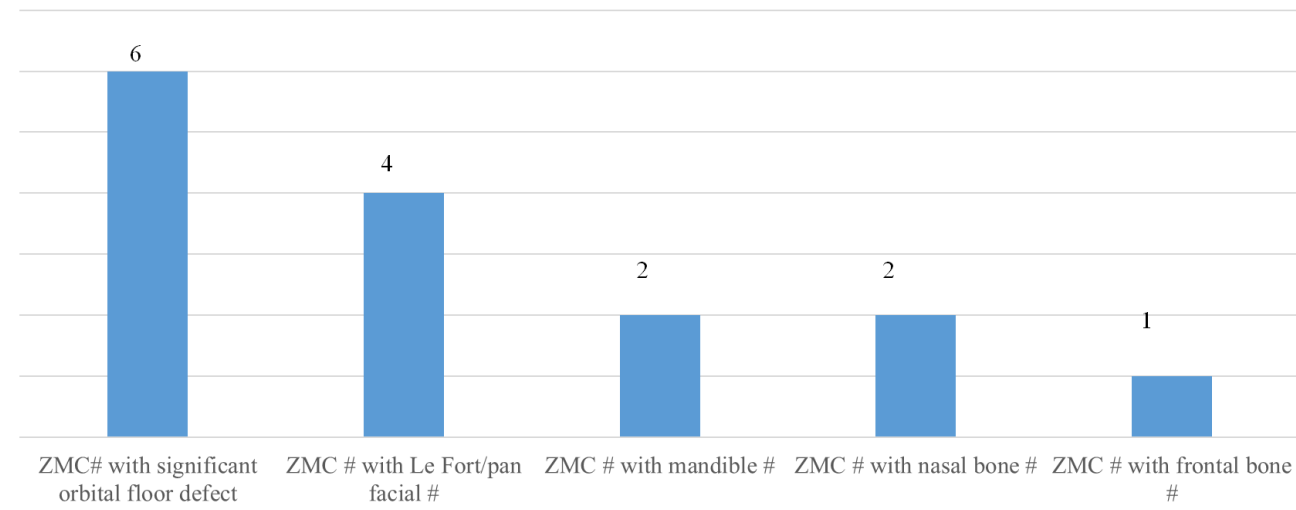

Figure 7. Bar chart showing the number associated maxillofacial injuries in our ZMC fracture cohort of 2016

Of those patients who had a ZMC fracture and associated maxillofacial injuries (20\%, $n=11)$, there were six cases of significant orbital floor defect. Orbital floor defects that resulted in eyes signs were confirmed radiologically on CT scans and were considered significant. There were additional associated maxillofacial injuries including four cases of Le Fort fracture patterns, two mandible fractures, two nasal bone fractures, and one frontal bone fracture [Figure 7].

\section{Operations}

As shown in Table 1, there were a total of 39 open reduction internal fixation (ORIF) of ZMC fractures. Of these, $27(69 \%)$ cases were ORIF of ZMC fracture only, and in 12 cases (31\%) there were other associated procedures including orbital floor exploration $(10 \%, n=4)$, orbital floor repair $(7.5 \%, n=3)$, ORIF of Le Fort fractures $(5 \%, n=2)$, ORIF of Mandible and Le Fort fractures $(5 \%, n=2)$ and one nasal MUA $(2.5 \%)$.

In one case, a polytrauma patient who had surgery delayed by 49 days due to their concurrent injuries, it was not possible to reduce the ZMC fracture intra-operatively. A bone graft was therefore taken from the anterior maxillary sinus wall and secured to zygomatic body to aesthetically improve the cheek flattening. 
Table 1. Distribution of operative cases of ZMC fractures in 2016: including ORIF ZMC only, ORIF ZMC and associated operative procedures, and indirect reduction (Gillies and Keen's)

\begin{tabular}{ll}
\hline OPERATION & Number $(\boldsymbol{n}) \mathbf{,}(\mathbf{\%})$ \\
\hline ORIF & Total $=39$ \\
ORIF ZMC only & $27(69 \%)$ \\
ORIF ZMC + Orbital floor exploration & $4(10 \%)$ \\
ORIF ZMC + Orbital floor repair & $3(7.5 \%)$ \\
ORIF ZMC + ORIF Le Fort & $2(5 \%)$ \\
ORIF ZMC + ORIF Mandible + ORIF Le Fort & $2(5 \%)$ \\
ORIF ZMC + Nasal MUA & $1(2.5 \%)$ \\
BONE GRAFT & Total $=1$ \\
Not possible to reduce fracture intra-operatively & 1 \\
INDIRECT REDUCTION & Total $=13$ \\
Gillies lift & $11(85 \%)$ \\
Keen's & $2(15 \%)$ \\
\hline
\end{tabular}

ORIF: Open Reduction and Internal Fixation; ZMC: zygomatic complex

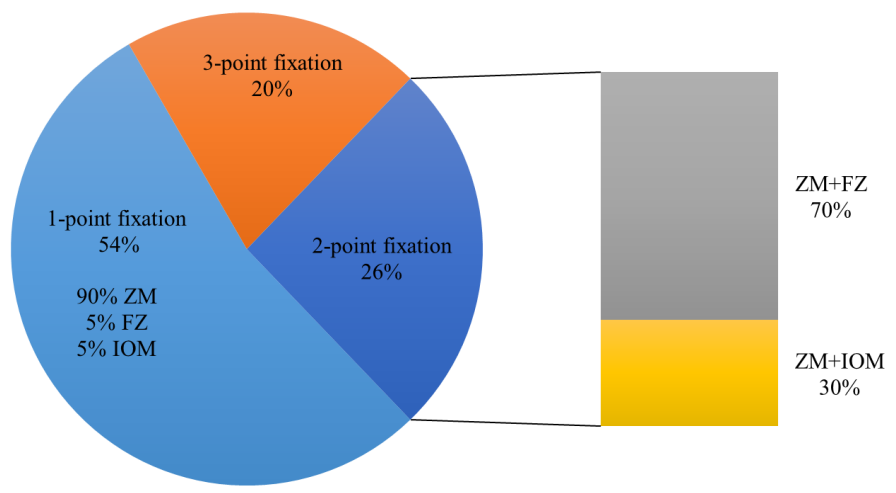

Figure 8. Chart illustrating the number of fixation points used and their location in all cases of ORIF ZMC fractures in the 2016 cohort $(n=39)$

All of the 13 isolated zygomatic arch fractures were treated with indirect reduction using Gillies $(85 \%, n=11)$ and Keen's $(15 \%, n=2)$ approaches.

\section{Points of fixation and access (incisions)}

Up to three anatomical points of fixation (plating) were used for ORIF of ZMC fractures: zygomaticomaxillary (ZM) buttress, frontozygomatic (FZ) suture and infraorbital margin (IOM). For FZ suture fixation, three types of incisions were used: upper blepharoplasty, lateral eyebrow, and existing scar. For IOM fixations, three types of incisions were used: subciliary, subtarsal and transconjunctival.

As previously mentioned, one ZMC fracture case in a polytrauma patient who had surgery delayed 49 days due to concurrent injuries was managed with a bone graft as is was not possible to reduce the ZMC fracture intra-operatively. We shall therefore use the remaining 39 ORIF ZMC cases to analyse points of fixations and surgical access used.

Of these 39 cases of ORIF ZMC (with or without other associated fixations): $54 \%(n=21)$ had one-point fixations, 26\% $(n=10)$ had two-point fixations, and 20\% $(n=8)$ had three-point fixations [Figure 8]. Of the one-point fixations $(n=21), 90 \%(n=19)$ had fixation at the ZM buttress, and in the remaining two cases, one had fixation at the FZ via a lateral eyebrow incision, and the other had fixation at the IOM via a transconjunctival incision. Of the two-point fixations $(n=10), 70 \%(n=7)$ had fixation at the ZM buttress and $\mathrm{FZ}$, and $30 \%(n=3)$ had fixation at the ZM buttress and IOM. 

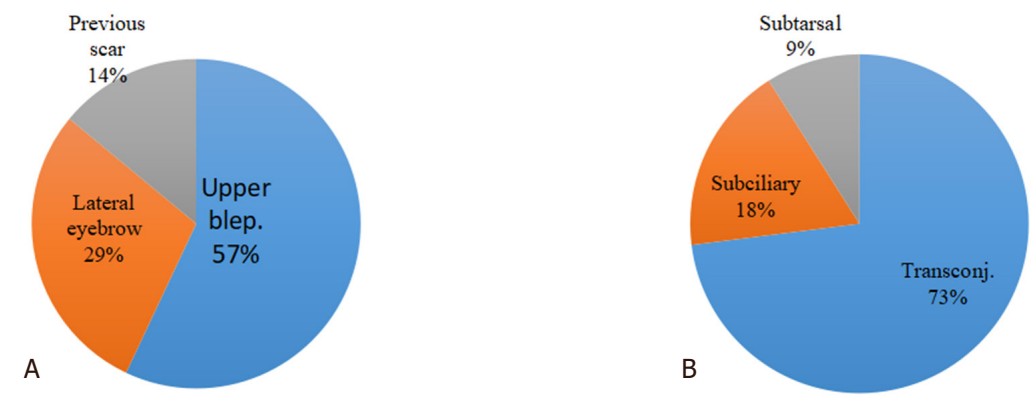

Figure 9. Graphs illustrating the type of incision used to access the frontozygomatic (FZ) suture (upper blepharoplasty, lateral eyebrow and previous scar (A); and the infraorbital margin (IOM) (transconjunctival, subciliary and subtarsal) (B)

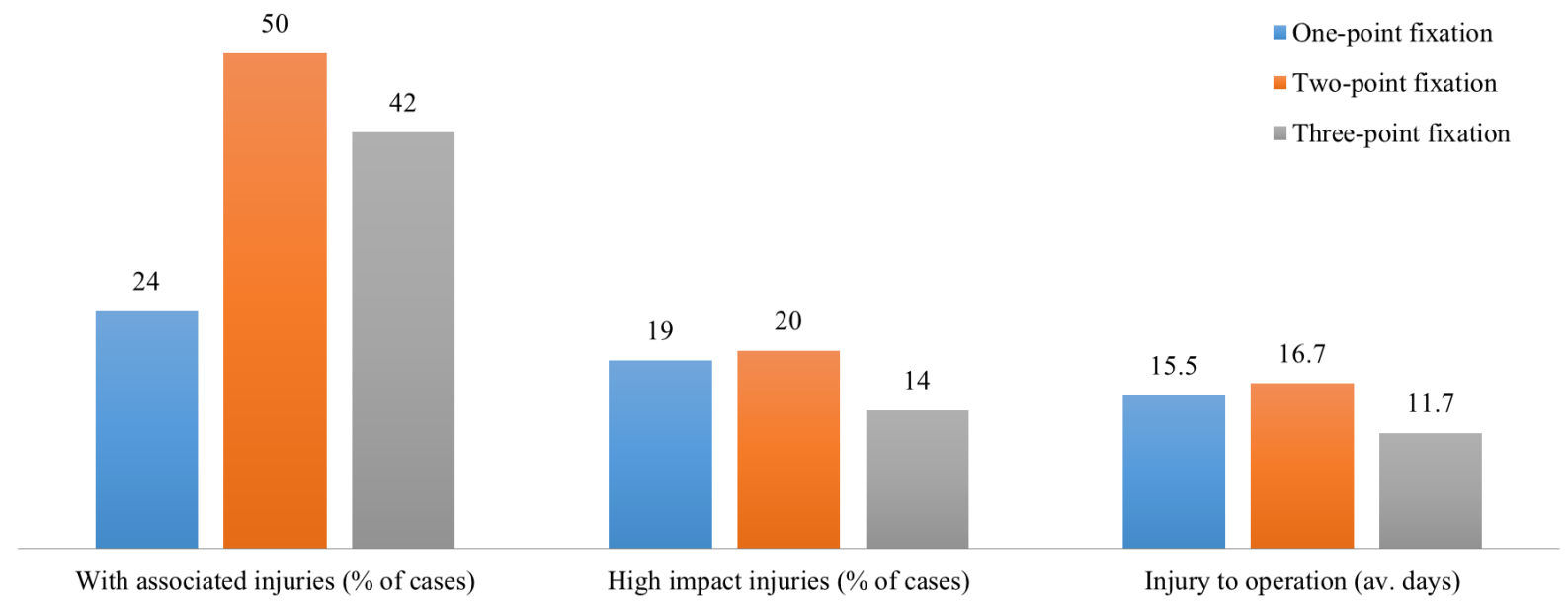

Figure 10. Graphs showing the association between number of fixation point versus: associated injuries, high impact injuries, and injury to operation time

Overall, 95\% $(n=37)$ of cases involved a ZM buttress fixation, all accessed through an intra-oral buccalsulcus incision. Furthermore, $41 \%(n=16)$ of cases involved an FZ fixation: one case for a one-point fixation, 7 cases for a two-point fixation, and 8 cases for a three-point fixation. Upper blepharoplasty incision was used in $56 \%(n=9)$ of FZ fixations, with $31 \%(n=5)$ via a lateral eyebrow incision, and $13 \%(n=2)$ via a previous scar [Figure 9]. Lastly, 31\% $(n=12)$ of cases involved an IOM fixation: one case for a one-point fixation, 3 cases for a two-point fixation, and 8 cases for a three-point fixation. Of the IOM fixations $(n=12)$, $75 \%(n=9)$ were via a transconjunctival incision, $17 \%(n=2)$ via a subciliary incision and $8 \%(n=1)$ via a subtarsal incision [Figure 9].

Number of fixation versus associated injuries, impact of injury, and injury to operation duration Of the two-point fixation cases $(n=10), 50 \%(n=5)$ had other associated maxilla-facial injuries, of the three-point fixation cases $(n=8), 42 \%(n=3)$ had other associated maxillofacial injuries. In the one-point fixation cases $(n=21)$, only $24 \%(n=4)$ had other associated facial injuries [Figure 10]. However, there was statistically significant correlation shown between the number of fixation points and presence of associated injuries $(P=0.52)$.

High impact injuries accounted for 19\% $(n=4)$ of one-point fixation cases, $20 \%(n=2)$ of two-point fixation cases and $14 \%(n=1)$ of three-point fixation cases [Figure 10]. RTCs, assaults from hard objects, or falls from a significant height (greater than $2 \mathrm{~m}$ ), were considered high impact injuries. There was no significant correlation found between the number of fixation points and the impact of injury. 
The average duration (days) between injury to operation was 15.5, 16.7 and 11.7, for one-, two- and threepoint fixations, respectively [Figure 10]. There was no significant correlation between the number of fixation points and duration from injury to operation.

\section{Immediate and long-term complications}

There were no immediate postoperative complications. As previously mentioned, in one case, a polytrauma patient who had surgery delayed by 49 days due to their concurrent injuries, it was not possible to reduce the $\mathrm{ZMC}$ fracture intra-operatively. A bone graft was therefore taken from the anterior maxillary sinus wall and secured to zygomatic body to aesthetically improve the cheek flattening from the ZMC fracture. The patient had an aesthetically satisfactory result and on subsequent outpatient follow-up the patient was pleased with their improved cheek contour from the bone graft. Another patient had delayed improvement of mouth opening, which subsequently resolved.

\section{Follow up}

Of the 53 surgically managed patients in our cohort, 4 (7.5\%) did not attend follow up and in 11 (21\%) patients follow up status was not documented. Twenty (38\%) patients were discharged from OMFS after their first outpatient follow up consultation, 13 (25\%) were discharged after their 2nd consultation, and $5(9 \%)$ after their 3 rd. All patients who attended follow up had satisfactory aesthetic and functional outcomes.

\section{DISCUSSION}

This article provides an overview of the epidemiology, aetiology, presentation, and management of surgicallytreated cases of ZMC fractures at our major trauma centre over a one year period.

The demographic data showed that the commonest age group (mode) presenting with ZMC fractures was 27 years of age, and the incidence was significantly greater (89\%) in men compared to women (11\%), which was in keeping with the current literature ${ }^{[1-4]}$. The commonest aetiology was interpersonal violence (53\%), followed by falls, RTCs and sport-related injuries. According to existing literature, aetiology is variable, with one study in Poland quoting assault as the most common, followed by RTC and other studies from Brazil and Amsterdam, showing RTCs to be most common, followed by assault ${ }^{[2,4,7,8]}$. These variations confirm that the aetiology of ZMC fractures are influenced by multiple factors, including geographical location, incidence of RTCs and socioeconomic trends. Within our demographics, the majority were low impact injuries (81\%), such as from punch-related assaults or mechanical falls $(<2 \mathrm{~m})$, compared to $15 \%$ related to RTCs, assaults from hard objects, or falls from a significant height $(>2 \mathrm{~m})$ which were considered as high impact injuries. We are unable to comment on the significance of alcohol or illicit drug use relating to injury due to inadequate documentation of this in patients' notes.

Of the 13 patients in our cohort with isolated zygomatic arch fractures, all were treated with indirect reduction using Gillies lift or Keen's approach. This was similar to a study in which 26 isolated zygomatic arch fractures were all managed with indirect reduction ${ }^{[4]}$.

Of the thirty-nine ZMC fractures in our cohort, all were treated with ORIF. This is in keeping with a study of $532 \mathrm{ZMC}$ fractures in which all were treated with $\mathrm{ORIF}^{[14]}$. However, in a retrospective study of 210 cases of ZMC fractures, $84 \%(n=177)$ patients had ORIF, whilst $16 \%(n=33)$ had closed reduction ${ }^{[4]}$. A survey answered by over 1600 ENT, OMFS and plastic surgeons that showed $81 \%$ would choose ORIF for ZMC fractures $^{[15]}$. None of the ZMC fractures in our cohort were treated with closed reduction.

Out of the $39 \mathrm{ZMC}$ fracture patients included in our study, 10\% $(n=4)$ underwent orbital floor exploration and $7.5 \%(n=3)$ underwent orbital floor repair. Our proportion of ORIF ZMC fractures undergoing associated 
orbital floor exploration was lower in comparison to a study of 72 patients with ZMC fractures, where 30\% of patients underwent orbital floor exploration ${ }^{[16]}$. Some centres carry out orbital floor exploration in cases of primary diplopia or evidence of comminuted ZMC fractures only ${ }^{[17]}$. Interestingly, as demonstrated in a survey involving facial reconstructive surgeons, it was shown that $35 \%$ would carry out an orbital floor exploration routinely ${ }^{[15]}$. The proportion of patients in our cohort undergoing orbital floor repair was also lower when comparing to the literature. In a study of 758 patients with ZMC fractures, where intraoperative CT imaging was used, $40 \%$ of patients underwent orbital floor repair, compared to $7.5 \%$ in our cohort, although intraoperative CT imaging is not used in our centre for ZMC fracture fixation ${ }^{[18]}$. Overall, these comparisons highlight the ongoing lack of consensus regarding the management of orbital floor defects in association with ZMC fractures.

Anatomically, although ZMC fractures will result in an orbital floor defect to a certain degree, not all cases warrant surgical exploration or repair of the orbital floor ${ }^{[18]}$. Orbital floor exploration and/or repair is often required in the presences of eye signs (enopthalmus, hypoglobus, diplopia, restricted eye movements) or a significant defect with or without ocular muscular entrapment seen on CT imaging. At present, aside from clinical judgment based on examination and imaging, there is no clear consensus or guideline to determine which cases of $\mathrm{ZMC}$ fractures require orbital floor exploration or repair. Further investigation to compare pre-operative clinical eye signs in ZMC fractures (enopthalmus, hypoglobus, diplopia, restricted eye movements), pre-operative orbital floor CT imaging, and the frequency of subsequent orbital floor exploration and repair between different centres would be useful to aid developing such a protocol.

Of the 39 cases that underwent ORIF, one-point fixations were the most popular ( $54 \%, n=21)$, followed by two-point fixations $(26 \%, n=10)$ and three-point fixations $(20 \%, n=8)$. Amongst the cases of one-point fixation, $90 \%(n=19)$ had fixation at the ZM buttress, $5 \%(n=1)$ at the FZ suture and $5 \%(n=1)$ at the IOM. Some literature supports the ZM buttress as the first choice for one-point fixations, with it providing sufficient stability, without the need for fixation at the FZ site, whilst some studies advocate FZ suture as the first choice, claiming that greater stability and immobilisation can be achieved at the FZ suture ${ }^{[4,11,13,19,20]}$. Of note, none of the literature reviewed advocated the IOM as the first choice location for one-point fixation.

Our incidence of one-point fixations was 54\%, which was higher compared to the literature, including Covington et al. ${ }^{[21]}$, who quoted that $30 \%-40 \%$ of $\mathrm{ZMC}$ fractures were adequately stabilised by one-point fixations, and Ellis and Kittidumkerng ${ }^{[22]}$, who quoted $31 \%^{[21,22]}$. A concern of one-point fixation can be that the zygoma may not be sufficiently stabilized against the rotational forces from the masseter upon mastication. In our cohort, there were no significant immediate or late post-operative complications, nor any long term aesthetic concerns of the malar area. We can therefore deduce that $54 \%$ of our cohort underwent successful ZMC stabilisation by one-point fixation. Of the two-point fixations, the most common sites of fixation were ZM buttress and FZ suture $(70 \%, n=7)$, followed by ZM buttress and IO rim $(30 \%, n=3)$. This was in keeping with a study of 210 surgically-managed ZMC fractures, in which similar anatomical locations for two-point fixations were used ${ }^{[4]}$.

All ZM buttress fixations in our cohort were accessed via an intraoral buccal sulcus incision, which was in keeping with the literature ${ }^{[4,9]}$. Given that this approach is intraoral, it has the advantage of avoiding any external facial scarring ${ }^{[23]}$. For FZ access $(n=16)$, upper blepharoplasty incision $(56 \%, n=9)$ was the most common, followed by lateral eyebrow $(31 \%, n=5)$ and 2 cases through an old scar or current laceration $(13 \%$, $n=2)$. For IOM access $(n=12)$, the most common incision was transconjunctival incision $(75 \%, n=9)$, followed by subciliary $(17 \%, n=2)$ and subtarsal $(8 \%, n=1)$. Some literature suggests that the incisions for infraorbital or orbital floor access carried the most complications, such as a study conducted on 180 patients showing complication rates of $1.5 \%$ and $14 \%$ for entropion in transconjunctival and subciliary incision, respectively, and a $3.4 \%$ incidence of hypertrophic scarring with subtarsal incisions ${ }^{[12,18]}$. Furthermore, the author of the 
same study stated a preference for subtarsal incisions for ZMC fractures and transconjunctival incision for isolated orbital floor fractures (blow-out fracture) ${ }^{[12]}$. Despite some reservations on the transconjunctival approach due to its close association with the eye, a study of 8 patients displayed no ocular complications (such as chemosis) ${ }^{[24]}$. Similarly, the 9 patients that underwent transconjunctival incision in our cohort did not present with any complications. The transconjunctival approach was also favoured by another study claiming that it has the advantages of both good intra-operative visualisation of the infraorbital rim, as well as having favourable aesthetic results for the patient ${ }^{[25]}$. In another study, Y-modification of a transconjunctival incision has been advocated for access the IOM and FZ area, with the advantage of potentially avoiding a second incision in the FZ area, although detailed knowledge of the lateral canthal anatomy is required and may increase operating time ${ }^{[2]}$. For FZ suture access, is in our cohort, the literature favoured the upper blepharoplasty incision over the lateral eyebrow incision, with the latter tending to show more scarring and less surgical access than the former, which often produces an inconspicuous scar that can only be seen when the eye lids are closed ${ }^{[26,27]}$.

Limitations of the study: ZMC fractures that were treated conservatively were not included in our data collection. It would be beneficial to elicit the epidemiology and presentations of conservatively treated cases compared to surgically treated cases. There was insufficient documentation of alcohol and illicit drug use to determine their possible link to ZMC fracture aetiology within our patient cohort. Additionally, further data collection of pre-operative imaging and fracture displacement measurements to ascertain the correlation between the radiographic findingsand each surgical procedure chosen would be useful. This would provide valuable information regarding the correlation between the type of ZMC fracture i.e., degree of displacement or comminution, and the subsequent choice of number of fixation points.

This study supports aspects of the current literature regarding the aetiology and surgical management of ZMC fractures. It has been shown that the aetiology of ZMC fractures does indeed vary with geographical areas and incidence of RTCs. At our centre, one-point fixation was the most popular technique for surgical stabilization of ZMC fractures, with the ZM buttress being the most popular choice. It is generally accepted that sufficient stability is obtained with one-point fixation when there is no comminution of the ZMC fracture, with two-point and three-point fixation providing increasing stability where necessary, and often based on fracture comminution and surgeon's preference ${ }^{[15]}$. The upper buccal sulcus incision is widely accepted as the best approach for ZM buttress fixation. For IOM access, the transconjunctival and subciliary incisions appear to be most popular within the literature, both having advantages of providing good intra-operative views, low incidence of ocular complications, and good aesthetic outcomes. Interestingly, our incidence of orbital floor exploration and orbital floor repair was lower than that reviewed in the literature. It would be of benefit to further investigate this, with the aim of developing a specific protocol for orbital floor exploration and repair based on pre-operative imaging and clinical signs.

Although there is some agreement amongst certain aspects of the surgical management of ZMC fractures, there is still an ongoing lack of consensus in many aspects, particularly with regards to the number and location of fixation points used and orbital floor exploration and repair. It appears that the surgeon's experience, training background, and preferences may play a significant role in contributing to and maintaining the variety of surgical approaches to ZMC fractures.

Further work in constructing a management protocol for ZMC fractures, alongside well-designed prospective research, would minimise the lack of consensus and optimise care for ZMC fracture patients.

\section{DECLARATIONS}

\section{Authors' contributions}

All authors contributed to the manuscript. 


\section{Availability of data materials}

All data can be found on King's College London Hospital NHS Foundation Trust Online Database.

\section{Financial support and sponsorship}

None.

\section{Conflicts of interest}

All authors declared that there are no conflicts of interest.

\section{Ethical approval and consent to participate}

Not applicable.

\section{Consent for publication}

Not applicable.

\section{Copyright}

(C) The Authors 2019.

\section{REFERENCES}

1. Venugopal MG, Sinha R, Menon PS, Chattopadhyay PK, Roy Chowdhury SK. Fractures in the maxillofacial region: a four year retrospective study. Med J Armed Forces India 2010;66:14-7.

2. Brasileiro BF, Passeri LA. Epidemiological analysis of maxillofacial fractures in Brazil: a 5-year prospective study. Oral Surg Oral Med Oral Pathol Oral Radiol Endod 2006;102:28-34.

3. Ribeiro Ribeiro AL, da Silva Gillet LC, de Vasconcelos HG, de Castro Rodrigues L, de Jesus Viana Pinheiro J, et al. Facial fractures: large epidemiologic survey in Northern Brazil reveals some unique characteristics. J Oral Maxillofac Surg 2016;74:2480.e1-12.

4. Forouzanfar T, Salentijn E, Peng G, van den Bergh B. A 10-year analysis of the "Amsterdam" protocol in the treatment of zygomatic complex fractures. J Craniomaxillofac Surg 2013;41:616-22.

5. McBride S, Barry T. Fractures of the zygomatic complex - a comprehensive review over 10 years of surgical management. Br J Oral Maxillofac Surg 2015;53:e72.

6. Dakir A, Muthumani T, Prabu NP, Mohan R, Maity A. One point fixation of zygomatic tripod fractures in the zygomatic buttress through Keen's intraoral approach: a review of 30 cases. J Pharm Bioallied Sci 2015;7:S238-41.

7. Calderoni DR, Guidi Mde C, Kharmandayan P, Nunes PH. Seven-year institutional experience in the surgical treatment of orbitozygomatic fractures. J Craniomaxillofac Surg 2011;39:593-9.

8. Bogusiak K, Arkuszewski P. Characteristics and epidemiology of zygomaticomaxillary complex fractures. J Craniofac Surg 2010;21:1018-23.

9. AOFoundation. AO Craniomaxillofacial (AOCMF). 2017. Available from: https://aocmf.aofoundation.org/Structure/about-aocmf/ Pages/about.aspx. [Last accessed on 17 May 2019]

10. Farber SJ, Nguyen DC, Skolnick GB, Woo AS, Patel KB. Current management of zygomaticomaxillary complex fractures: a multidisciplinary survey and literature review. Craniomaxillofac Trauma Reconstr 2016;9:313-22.

11. Habal MB. The orbits: it is less important what you put in than how you secure it. J Craniofac Surg 2010;21:965-6.

12. Ridgway EB, Chen C, Colakoglu S, Gautam S, Lee BT. The incidence of lower eyelid malposition after facial fracture repair: a retrospective study and meta-analysis comparing subtarsal, subciliary, and transconjunctival incisions. Plast Reconstr Surg 2009;124:1578-86.

13. Olate S, Lima SM Jr, Sawazaki R, Moreira RW, de Moraes M. Surgical approaches and fixation patterns in zygomatic complex fractures. J Craniofac Surg 2010;21:1213-7.

14. Ji SY, Kim SS, Kim MH, Yang WS. Surgical methods of zygomaticomaxillary complex fracture. Arch Craniofac Surg 2016;17:206-10.

15. Baylan JM, Jupiter D, Parker WL, Czerwinski M. Management of zygomatic fractures: a national survey. J Craniofac Surg 2016;27:1571-5.

16. Shumrick KA, Kersten RC, Kulwin DR, Smith CP. Criteria for selective management of the orbital rim and floor in zygomatic complex and midface fractures. Arch Otolaryngol Head Neck Surg 1997;123:378-84.

17. Kovacs AF, Ghahremani M. Minimization of zygomatic complex fracture treatment. Int J Oral Maxillofac Surg 2001;30:380-3.

18. Ellis E 3rd, Perez D. An algorithm for the treatment of isolated zygomatico-orbital fractures. J Oral Maxillofac Surg 2014;72:1975-83.

19. Davidson J, Nickerson D, Nickerson B. Zygomatic fractures: comparison of methods of internal fixation. Plast Reconstr Surg 1990;86:25-32.

20. Kim JH, Lee JH, Hong SM, Park CH. The effectiveness of 1-point fixation for zygomaticomaxillary complex fractures. Acta Otolaryngol 2012;138:828-32. 
21. Covington DS, Wainwright DJ, Teichgraeber JF, Parks DH. Changing patterns in the epidemiology and treatment of zygoma fractures: 10-year review. J trauma 1994;37:243-8.

22. Ellis E 3rd, Kittidumkerng W. Analysis of treatment for isolated zygomaticomaxillary complex fractures. J Oral Maxillofac Surg 1996;54:386-400; discussion 400-1.

23. Kim ST, Go DH, Jung JH, Cha HE, Woo JH, et al. Comparison of 1-point fixation with 2-point fixation in treating tripod fractures of the zygoma. J Oral Maxillofac Surg 2011;69:2848-52.

24. Kumar S, Shubhalaksmi S. Clinical outcome following use of transconjunctival approach in reducing orbitozygomaticomaxillary complex fractures. Contemp Clin Dent 2016;7:163-9.

25. Manganello-Souza LC, Rodrigues de Freitas R. Transconjunctival approach to zygomatic and orbital floor fractures. Int J Oral Maxillofac Surg 1997;26:31-4.

26. Rajkumar K, Mukhopadhyay P, Sinha R, Bandyopadhyay TK. 'Y' modification of the transconjunctival approach for management of zygomatic complex fractures: a prospective analysis. J Maxillofac Oral Surg 2016;15:45-51.

27. Fonesca RJ, Walker RV, Betts NJ, Barber HD, Powers MP. Oral and Maxillofacial Trauma. 3rd ed. St Louis: Elsevier Saunders; 2005. 